\title{
Forecasting method of clean energy development potential considering sector coupling
}

\author{
Yu Zhu ${ }^{1}$, Weijie Shen ${ }^{2,}$, Changhao Zhang ${ }^{3}$, Yangyang Ge ${ }^{1}$, Danting Cai ${ }^{3}$ \\ ${ }^{1}$ State Grid Liaoning Electric Power Research Institute, Shenyang 110006, China. \\ ${ }^{2}$ School of Economy and Management, North China Electric Power University, Beijing 102206, China. \\ ${ }^{3}$ School of Electrical and Electronic Engineering, North China Electric Power University, Beijing 102206, China.
}

\begin{abstract}
Sector coupling includes not only the coupling within energy sectors such as electricity, heat and gas, but also the coupling between energy sector and transportation sector, construction sector and industrial sector. This article introduces a method for measuring the development potential of clean energy based on sector coupling. First, the analysis model of electric energy substitution potential is constructed, the analysis object is determined, and the analysis object is quantified. On the basis of the definition of clean energy development potential, an IPAT model for electric energy substitution is constructed to realize the comprehensive evaluation of clean energy. Secondly, based on Markov theory to realize the sub-path calculation of the development potential of clean energy. Finally, based on the IPAT model and the decoupling theoretical model, this paper sets up three different alternative scenarios to make a more comprehensive prediction and analysis of the medium and long-term clean energy development potential, and applies the introduced measurement methods to the calculation of China's clean energy development potential. The results show the effectiveness of the algorithm in the calculation of the development potential of clean energy based on sector coupling.
\end{abstract}

\section{Introduction}

Energy is the foundation of human survival and development, an important driving force for the industrial revolution, and a strong support for social and economic operations [1].The literature [2, 3, 4] all pointed out that the development and utilization of clean energy is the current development trend of the world's energy industry. In the literature [5], it is pointed out that China will adopt relevant measures to accelerate the development and utilization of clean energy. Literature [6] pointed out that under the current situation, increasing the proportion of renewable energy use and achieving clean energy is the general trend, and there is huge potential for cross- sector energy coupling.

Sector coupling includes not only the internal coupling of electricity, heat, gas and other energy industries, but also the coupling between the energy sector and the transportation, construction, and industrial sectors. Transportation systems, building energy, and industrial systems are all important loads of the energy system. With the development of the transportation system in the direction of electrification and intelligence, promoting the integrated and coordinated development of energy systems and electrified transportation systems will be the inevitable trend of economic efficiency, safe and stable operation and sustainable development of cities. As the construction sector develops towards green and lowcarbon, realizing the coupled development of the energy- building sector is a necessary means to improve energy efficiency and build green buildings. Optimizing and system integration in a larger scope and scale is of positive and far-reaching significance. With the development of industrial production in the direction of electrification and automation, the interaction between industrial systems and transportation systems has become increasingly significant. Promoting the integrated and coordinated development of the energy system and industry is the trend of the coordinated development of the industrial system and the energy industry.

\section{Forecasting method of clean energy development potential considering sector coupling}

\subsection{The basic process of potential calculation}

To conduct a mid- and long-term analysis of the development potential of clean energy, we must first determine the object of analysis, quantify the object of analysis, and construct relevant basic models. Since the development potential of clean energy cannot be measured solely by the amount of electricity demand, the amount of electric energy substitution is first defined to quantify the progress of the development of clean energy. Build an IPAT model on the development of clean energy based on the definition of the development potential of

\footnotetext{
* Corresponding author: shenweijie233@163.com
} 
clean energy. Then introduce the decoupling theory as the basis of multi-scenario construction and parameter setting. Finally, the scenario analysis method and the econometric analysis method are combined to realize the prediction and analysis of the development potential of clean energy. The basic process of potential analysis is shown in Figure 1.

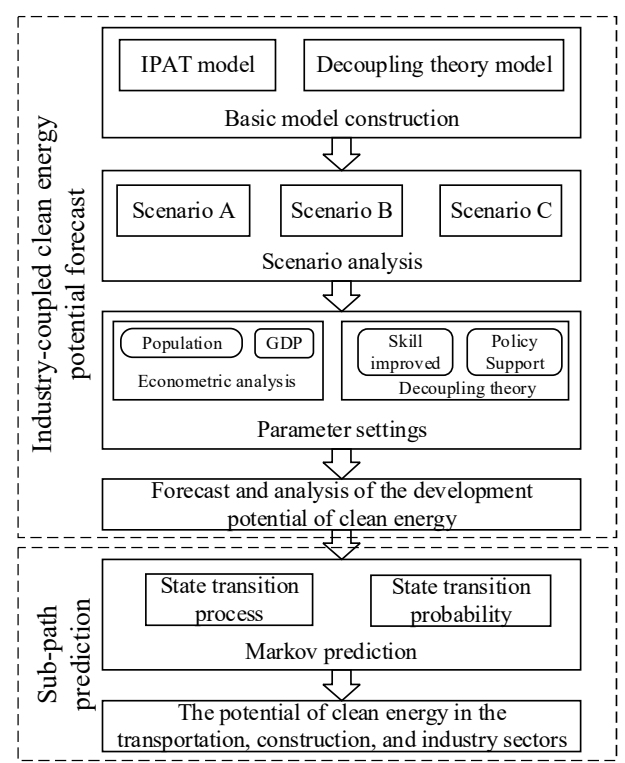

Figure 1. Flow chart of analysis of the development potential of clean energy.

\subsection{Model construction of the development potential of clean energy based on IPAT}

In order to realize the quantitative calculation of the development potential of clean energy, the amount of electric energy substitution is defined in this article as an objective basis for analyzing the development potential of clean energy. Set the base year TB. If the terminal energy consumption pattern is maintained at the base year level, the proportion of electric energy in the terminal energy is the same as that in the base year, and the increase in electric energy consumption in year $t$ compared to the baseline electric energy consumption is defined as the amount of electric energy substitution:

$$
D_{e, t}=\left(\mathrm{Y}_{e, t}-\frac{Y_{e, T_{B}}}{Y_{T_{B}}} . Y_{t}\right)
$$

In the formula: $D_{e, t}$ is the amount of electric energy substitution; $\mathrm{Y}_{e, t}$ is the actual electric energy consumption in year $\mathrm{t}$; $Y_{t}$ is the total terminal energy consumption in year $t$.

Barry Kemenler, Paul Ralph Ehrlich, and JP Holder jointly proposed the IPAT model in the 1970s[7].The IPAT model is a well-known formula that uses population $(\mathrm{P})$, affluence $(\mathrm{A})$, and technology level $(\mathrm{T})$ to evaluate the pressure of the environment. Its expression is:

$$
I=P \times A \times T
$$

The establishment of the IPAT model is to study the impact of population on environmental changes and reflect the relationship between changes in the environment affected by the three factors of population, wealth and technology. Electric energy substitution is also affected by the comprehensive effect of population, economic growth and technological level. Population growth, economic improvement and the development of related technologies will all promote the substitution of electric energy for terminal energy. Among them, the technological progress of electric energy substitution can be reflected in the intensity of electric energy consumption and the proportion of electric energy in the terminal energy consumption. As the technologies related to electric energy substitution become more mature, the intensity of electric energy consumption and the proportion of electric energy in terminal energy consumption will increase accordingly.

Therefore, by decomposing the above relational expressions, the expression of the IPAT model for electric energy substitution can be obtained:

$$
C_{t}=P_{t} \times A_{t} \times\left(Y_{\mathrm{e}} / G\right)_{t} \times\left(D_{\mathrm{e}} / Y_{\mathrm{e}}\right)_{t}
$$

In the formula: $C t$ represents the total amount of electric energy replacement in year $t ; P_{t}$ represents the total population in year $t$; $A_{t}$ represents the per capita GDP level in year $t ; D_{\mathrm{e}}$ represents the amount of terminal electrical energy replacement; $Y_{\mathrm{e}}$ represents the terminal electrical energy consumption; $G$ represents the GDP value; $\left(Y_{\mathrm{e}}\right.$ $/ G)_{t}$ represents the intensity of terminal power consumption in $t ; D_{\mathrm{e}} / Y$ represents the ratio of electric energy substitution to the total terminal electric energy consumption, that is, the electric energy terminal substitution structure.

Comparing the electric energy substitution situation in year $t$ with the base year, suppose the population growth rate is $\alpha$, the average annual growth rate of per capita GDP is $\beta$, and the increase rate of terminal power consumption intensity is $\gamma$, which represents the degree of electrification of terminal energy consumption, that is, the degree of technological progress. Use $\delta$ to indicate the rate of change of the proportion of electric energy substitution in the terminal's electric energy consumption, that is, the rate of change of the electric energy terminal's substitution structure.

Since the government-led industrial structure optimization and adjustment and the consumptionoriented role of clean energy are the main factors that affect the change of the terminal energy substitution structure, and the terminal clean energy mainly refers to electric energy, so $\delta$ can be regarded as the strength of the government's support for electric energy substitution. In the model, in year $t$, the relationship between various factors about the base year is as follows:

$$
\begin{gathered}
P t=P 0 \times(1+\alpha) \\
A t=A 0 \times(1+\beta) \\
(Y \mathrm{e} / G) t=(Y \mathrm{e} / G) 0 \times(1+\gamma) \\
(D \mathrm{e} / Y) t=(D \mathrm{e} / Y) 0 \times(1+\delta)
\end{gathered}
$$

In the formula: $\mathrm{Pt}$ and $\mathrm{P} 0$ are the total population in year $\mathrm{t}$ and the base year respectively; At and A0 are the GDP per 
capita in year $t$ and the base year respectively; De is the replacement amount of terminal electric energy; Ye is the consumption of terminal electric energy.

Therefore, the change in the total amount of electric energy substitution in the base year in year $t$ can be expressed as:

$$
C=C \times[(1+\alpha) \times(1+\beta) \times(1-\gamma) \times(1+\delta)] t
$$

In the formula, $\mathrm{Ct}$ and $\mathrm{C} 0$ are the total electric energy substitution in year $t$ and the base year, respectively.

\subsection{Basis for multi-scenario setting}

Since the increase in the proportion of terminal power consumption can promote economic growth, that is, economic growth and the degree of power substitution have a certain correlation, which can be measured by the decoupling theory. The degree of decoupling of development can be used as an important evaluation basis for technological progress and policy guidance. $X$ indicates the degree of decoupling. Here, the rate of change of GDP per capita $\lambda$ and the rate of change of electric energy substitution $\mu$ are used to express the degree of dependence between economic growth and terminal electric energy substitution:

$$
\chi=\mu / \lambda
$$

When the degree of decoupling $>1$, it indicates that the growth rate of electric energy substitution is greater than the economic development speed, the speed of electric energy substitution is fast, and the economic growth depends on the degree of electric energy substitution, and it is in the development stage of not decoupling. When the degree of decoupling=1, the growth rate of electric energy substitution is lower than the economic growth rate or consistent with the economic development rate. Economic growth does not completely or rarely depends on the degree of electric energy substitution, and the process of economic growth and electric energy substitution is in a decoupling stage $(1+\beta) /(1+\gamma) \times(1+\delta)$ $>1$ indicates that the speed of economic development is faster than the process of electric energy substitution, and the implementation of electric energy substitution is highly dependent on economic development. At this time, the degree of technological advancement and government support has made little effort to promote the substitution of electric energy, and decoupling from economic development cannot be achieved. $(1+\beta) /$ $(1+\gamma) \times(1+\delta)>=1$ indicates that the speed of economic development is basically the same as the process of electric energy substitution, and the popularization and application of electric energy substitution is not completely dependent on economic development. At this time, technological progress is the main factor to promote the substitution of electric energy, and decoupling from economic development can be achieved. $(1+\beta) /(1+\gamma) \times(1+\delta)<1$ indicates that the speed of electric energy substitution is greater than the speed of economic development, and the promotion of electric energy substitution no longer depends on economic development. At this time, in addition to the impact of technological progress on the development of electric energy substitution, the support of relevant policies for electric energy substitution also promotes electric energy substitution, and the absolute decoupling from economic development has truly been realized.

\subsection{Basis for sub-path calculation}

There are many coupling interaction variables between the energy sector and transportation sector, construction sector, industry sector and other sectors, and there is a certain degree of time and space correlation. Existing research is mostly based on the integrated system composed of energy and a single sector, such as the integrated system of the energy and transportation sector. The integration with multiple sectors will make the complexity of the model and the difficulty of solving it drastically increase, so it is necessary to adopt a better method to deal with changes in complex systems. The Markov method [8] is a probabilistic method for predicting the state of the system. It can predict the state of the system at various times in the future based on the current state of the system, and can effectively deal with the problem of calculating the potential of clean energy in the transportation sector, construction sector, and industrial sector routes under the coupling of sectors.

(1) State, state transition process.

In Markov prediction, state refers to a certain result of a certain event at a certain moment (or period). Generally speaking, as the researched event and its predicted target are different, the state can be divided in different ways. For example, there are "child", "juvenile", "youth", "middle-aged", "old age" and other states in the population system forecast.

The state transition process refers to the transition from one state to another during the development of an event. The state transition that the development of the system changes with the change of time, or the relationship between state transition and time, is called the state transition process, referred to as the process.

(2) State transition probability and state transition probability matrix.

In the development process of the system, the possibility of starting from a certain state and transferring to another state at the next moment is called the state transition probability. According to the definition of conditional probability, the state transition probability $P\left(E_{i} \rightarrow E_{j}\right)$ from state $E_{i}$ to state $E_{j}$ is the conditional probability $P\left(E_{j} / E_{i}\right)$, namely:

$$
P\left(E_{i} \rightarrow E_{j}\right)=P\left(E_{j} / E_{i}\right)=P_{i j}
$$

Assuming that a certain predicted system has $\mathrm{n}$ possible states, mark $P_{i j}$ as the state transition probability from state $E_{i}$ to state $E_{j}$, and make a matrix: 


$$
P=\left[\begin{array}{cccc}
P_{11} & P_{12} & \ldots & P_{1 n} \\
P_{21} & P_{22} & \ldots & P_{2 n} \\
\ldots & \ldots & \ldots & \ldots \\
P_{n 1} & P_{n 2} & \ldots & P_{n n}
\end{array}\right]
$$

Then $\mathrm{P}$ is the state transition probability matrix.

(3) State probability.

The state probability $\pi_{j}(k)$ represents the probability that the event is in the state $E_{i}$ at the kth moment after k times of state transitions under the condition that the initial state is known. According to the nature of probability, there are obviously:

$$
\sum_{j=1}^{N} \pi_{j}(k)=1
$$

Starting from the initial state, the state transition process of reaching the state $E_{j}$ after $k$ times of state transitions can be regarded as first reaching state $E_{i}(i=1,2, \ldots, n)$ after $(k-1)$ state transitions, and then reaching state $E_{j}$ through one state transition from $E_{i}$. According to the no aftereffect of Markov process and the Bayes conditional probability formula, there are:

$$
\pi_{j}(k)=\sum_{i=1}^{n} \pi_{i}(k-1) P_{i j}(j=1,2, \ldots n)
$$

If the row vector $\pi_{j}(k)=\left[\pi_{1}(k), \pi_{2}(k), \ldots \pi_{n}(k)\right]$ is recorded, the recursive formula for successively calculating the state probability can be obtained from the formula (13):

$$
\left\{\begin{array}{c}
\pi(1)=\pi(0) P \\
\pi(2)=\pi(1) P=\pi(0) P^{2} \\
\cdots \\
\pi(k)=\pi(k-1)=\pi(0) P^{k}
\end{array}\right.
$$

In (14), $\pi(0)$ is the initial state probability vector.

According to the above analysis, if the initial state of an event at the 0th moment is known, the probability of the event being in various possible states at the $k$ th moment after $k$ state transitions can be obtained by the recursion formula (14) (i.e. $\pi(k)$ ). Thus, the state probability prediction of the event at the $k$ th moment is obtained, that is, the potential calculation of the sub-path of clean energy in the transportation sector, construction sector and industrial sector under the coupling of sectors is realized.

\section{Case analysis}

In order to make a more comprehensive prediction and analysis of the development potential of clean energy in the medium and long term, this paper sets three different alternative scenarios based on the IPAT model and the decoupling theoretical model to provide support for the analysis of the development potential of clean energy based on different paths.
Among them, scenario A is based on the existing electric energy substitution development, and does not consider the effect of technological progress and policy support on electric energy substitution, that is, electric energy substitution and economic development are not decoupled. Scenario B introduces the promotion of technological substitution on the basis of the development of existing electric energy substitution, and realizes the decoupling of electric energy substitution and economic development. Scenario C Increases the driving force of policy support for electric energy substitution on the basis of scenario B, and realizes the complete decoupling of electric energy substitution and economic development. Predict the parameters of population, economic development, electric energy substitution technology level and related policy support under scenario A, scenario B and scenario C.

1) Population parameters. With reference to the predictions and judgments of the "National Population Development Strategy Research Report", "Population and Social Development Report 2021" and other relevant plans, and a comprehensive analysis of the changes in China's future population growth rate, the population growth rate from 2021 to 2025 is expected to be $2.4 \%$, the population growth rate from 2026 to 2030 is $2.2 \%$, the population growth rate from 2031 to 2035 is $1.3 \%$, the population growth rate from 2036 to 2040 is $1.3 \%$, the population growth rate from 2041 to 2045 is $3.1 \%$, and the population growth rate from 2046 to 2050 is $3.1 \%$. Set population parameters based on the above data.

2) Economic parameters. Economic growth has a certain degree of influence on terminal power consumption. According to the forecast of the Chinese Academy of Social Sciences "China's Economic Situation Analysis and Forecast Blue Book in 2021", China's economic growth rate in 2016 will be between $6.6 \%$ and $6.8 \%$. By 2030 , the economic growth rate will gradually slow down due to structural issues such as population aging. At the same time, the Economist Intelligence Unit report pointed out that the global economic growth in 2050 will not decline sharply compared with its historical level, but economic growth will slow down after 2030. Based on the actual GDP in 2021, it is estimated that $5.4 \%$ in 2021 $2025,4.1 \%$ in $2026-2030,3.8 \%$ in $2031-2035,3.75 \%$ in 2036-2040, and $3.5 \%$ in 2041-2045. From 2046 to 2050, it is $3.4 \%$.

3) Technical level. Assume that in scenario A, the development of electric energy substitution related technologies stagnates, the terminal electric energy demand remains at the current level, and the terminal electric energy consumption intensity remains unchanged. The electric energy substitution process is not decoupled from economic development due to the lack of technological advancement. The application field of electric energy substitution technology will be expanded on a large scale in 2021. Small coal-fired boilers are gradually being replaced by electric boilers, electric boilers + heat pumps, electric boilers + gas boilers, etc. Heating technologies such as carbon crystals, electric heating films, and heating cables are gradually being used in daily life. Related technologies such as military port shore power and railway electrification have also been applied on a large scale. It is assumed that electric energy 
substitution technology develops in coordination with economy; the demand for terminal power increases greatly with the development of power substitution technology; the consumption intensity of terminal electric energy increases, and the growth level is consistent with the economic development level of each stage, so that the decoupling degree of electric energy substitution and economic development is approximately 1 . In scenario C, the technical level of electric energy substitution at all stages is consistent with scenario B.

4) Policy support intensity. It is assumed that in scenarios $\mathrm{A}$ and $\mathrm{B}$, the government does not provide policy support and related interference in the work related to electric energy substitution, and the electric energy terminal substitution structure maintains stable development. Without government intervention, electricity substitution and the economy have not been decoupled or completely decoupled, and the degree of decoupling is not greater than one. Scenario $\mathrm{C}$ is based on China's work plans and goals related to electric energy substitution, increasing the government's support for electric energy substitution, enhancing the ability of electric power terminal substitution, changing the substitution structure, and greatly increasing the development potential of clean energy.

Since 2015, the government has issued 226 support policies to encourage electric energy substitution, guiding the society to actively choose electric energy, and gradually eliminating high-polluting and low-efficiency energy use methods. Since electric energy substitution can respond well to the green development requirements of China's 13th Five-Year Plan, and has been written into The State Council's Action Plan for Air Pollution Prevention and control and other important documents, many provinces and cities have also included the work of electric energy substitution in their 13th Five-Year Plan. Therefore, it is expected that the government's promotion of electric energy substitution will enter a peak period in the next 5 years. Thanks to the national policy guidance and the active efforts of the State Grid Corporation of China, in 2015, compared with 2014, the amount of electricity replacement increased by nearly 25 billion $\mathrm{kW}$ $\mathrm{h}$, with a growth rate of 0.49 . It is expected that policy support will continue to increase significantly from 2016 to 2020 until the level of technological progress related to electric energy substitution stabilizes, the field of electric energy substitution has been fully expanded, and the structure of electric energy terminal substitution has stabilized. It is estimated that after 2040, the work of electric energy substitution can be carried out well without government support. The economy of electric energy substitution makes the society actively choose electric energy as the main energy consumption of the terminal.

In addition to the promotion of electric energy substitution by technological and economic development, increase the government's encouragement and support for electric energy substitution related work, so that electric energy substitution and economic development are completely decoupled, and the development of electric energy substitution does not need to rely on economic development.

According to the decoupling theory, the population, per capita GDP, technological progress and policy support parameter settings under scenario A, scenario B and scenario $\mathrm{C}$ are used to predict the development and change of the end-use energy structure, as shown in the following table. Among them: $\alpha$ is the annual growth rate of the population; $\beta$ is the annual growth rate of GDP per capita; $Y$ is the growth rate of the level of electric energy substitution technology; $\sigma$ is the rate of change of policy support.

Table 1. Basic parameters of scenario analysis.

\begin{tabular}{cccccc}
\hline Year & Scenario & $\alpha / \%$ & $\beta / \%$ & $\gamma / \%$ & $\sigma / \&$ \\
\hline \multirow{2}{*}{$2016-2020$} & A & 0.35 & 6.00 & 0.00 & 0.0 \\
& B & 0.35 & 6.00 & 5.71 & 0.0 \\
& C & 0.35 & 6.00 & 5.71 & 4.5 \\
\hline \multirow{2}{*}{$2021-2025$} & A & 0.24 & 5.40 & 0.00 & 0.0 \\
& B & 0.24 & 5.40 & 5.54 & 0.0 \\
& C & 0.24 & 5.40 & 5.54 & 4.1 \\
\hline \multirow{2}{*}{$2026-2030$} & A & 0.21 & 4.10 & 0.00 & 0.0 \\
& B & 0.21 & 4.10 & 4.93 & 0.0 \\
& C & 0.21 & 4.10 & 4.93 & 3.2 \\
\hline \multirow{2}{*}{$2036-2040$} & A & -0.13 & 3.80 & 0.00 & 0.0 \\
& B & -0.13 & 3.80 & 4.30 & 0.0 \\
& C & -0.13 & 3.80 & 4.30 & 2.6 \\
\hline \multirow{2}{*}{$2041-2045$} & A & -0.13 & 3.75 & 0.00 & 0.0 \\
& B & -0.13 & 3.75 & 3.43 & 0.0 \\
& C & -0.13 & 3.75 & 3.43 & 2.1 \\
\hline \multirow{2}{*}{$2046-2050$} & A & -0.31 & 3.50 & 0.00 & 0.0 \\
& B & -0.31 & 3.50 & 3.21 & 0.0 \\
& C & -0.31 & 3.50 & 3.21 & 1.8 \\
\hline & A & -0.31 & 3.40 & 0.00 & 0.0 \\
& B & -0.31 & 3.40 & 2.84 & 0.0 \\
& C & -0.31 & 3.40 & 2.84 & 1.4 \\
\hline
\end{tabular}

Table 2. China's total electricity consumption by sector.

\begin{tabular}{ccccc}
\hline $\begin{array}{c}\text { Power } \\
\text { consumption }\end{array}$ & $\begin{array}{c}\text { Total } \\
(\mathrm{kWh})\end{array}$ & $\begin{array}{c}\text { Industry } \\
\text { sector } \\
(\mathrm{kWh})\end{array}$ & $\begin{array}{c}\text { Construction } \\
\text { sector(kWh) }\end{array}$ & $\begin{array}{c}\text { Transportation } \\
\text { sector(kWh) }\end{array}$ \\
\hline 2020year & 77091 & 51530 & 1061 & 1822 \\
2019 year & 74866 & 50698 & 991 & 1752 \\
2018 year & 71508 & 49094 & 887 & 1608 \\
2017 year & 65914 & 46052 & 789 & 1418 \\
2016 year & 61205 & 42996 & 725 & 1251 \\
2015 year & 58020 & 41550 & 698 & 1125 \\
2014 year & 57829 & 42248 & 721 & 1059 \\
2013 year & 54203 & 39236 & 675 & 1000 \\
2012 year & 49762 & 36232 & 608 & 915 \\
2011 year & 47000 & 34691 & 571 & 848 \\
2010 year & 41934 & 30871 & 483 & 734 \\
2009 year & 37032 & 26854 & 421 & 617 \\
2008 year & 34541 & 25388 & 367 & 571 \\
2007 year & 32711 & 24290 & 309 & 531 \\
2006 year & 28588 & 21267 & 271 & 467 \\
2005 year & 24940 & 18521 & 233 & 430 \\
2004 year & 21971 & 16424 & 202 & 449 \\
2003 year & 19031 & 14169 & 179 & 406 \\
2002 year & 16465 & 12402 & 154 & 303 \\
2001 year & 14723 & 10944 & 154 & 309 \\
\hline
\end{tabular}

Through calculations, the development potential of clean energy under sector coupling will show a substantial growth trend before 2030 under scenario A, scenario B, and scenario $C$, as shown in Figure 2. Among them, the growth rate of clean energy development potential under scenario A is smaller than scenario B and scenario C. This 
is because scenario A does not have technological progress and related policies to promote the replacement of electric energy. In scenario $\mathrm{C}$, due to the increase of government support for electric energy substitution related work, the growth rate of clean energy development potential under this scenario is greater than that in scenario B. Between 2030 and 2040, the development potential of clean energy will tend to grow steadily. Until 2045 , as the alternative field is close to saturation, the development potential of clean energy will gradually stabilize and there will be no substantial growth.

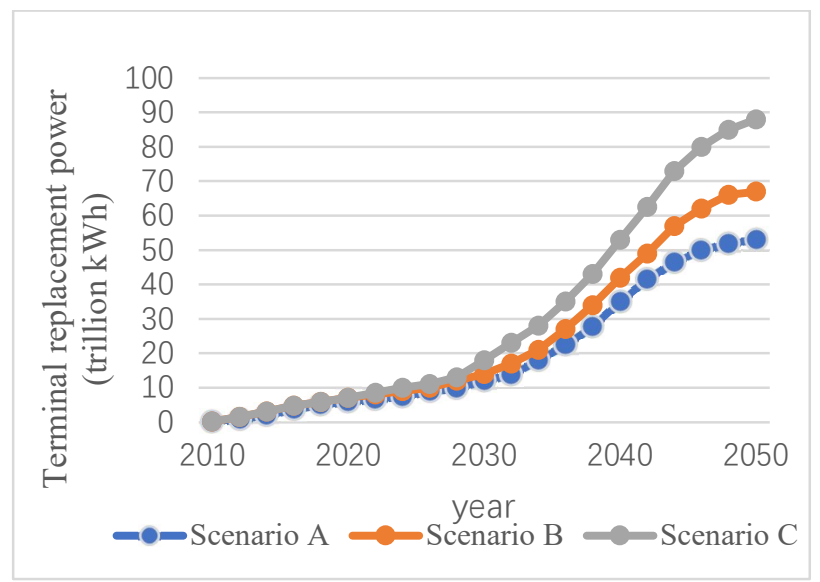

Figure 2. Forecast of clean energy development potential under various scenarios.

Compared with scenario A, the electric energy substitution process under scenario $\mathrm{B}$ and scenario $\mathrm{C}$ is significantly greater than scenario A. The increased policy support in Scenario C has a particularly significant effect on the promotion of electric energy substitution, and it is much higher than in Scenario B, which only relies on technological progress to promote electric energy substitution. In Figure 4, in 2020, 2030, 2040 and 2050, Scenario B will reach clean energy development potential of 480 million $\mathrm{kWh}, 1.71$ billion $\mathrm{kWh}, 4.39$ billion $\mathrm{kWh}$ and 6.5 billion $\mathrm{kWh}$, respectively. Compared with the clean energy development potential under Scenario A, the alternative electric energy is increased by $8.13 \%, 13.96 \%$, $18.43 \%$ and $22.05 \%$, respectively. In Scenario C, due to increased policy support, the development potential of clean energy will grow more rapidly. Compared with scenario A, this scenario will increase the alternative electric power by 520 million $\mathrm{kWh}, 2.03$ billion $\mathrm{kWh}$, 5.55 billion $\mathrm{kWh}$, and 8.61 billion $\mathrm{kWh}$, respectively, and the increase rate reaches $17.83 \%, 35.52 \%, 49.87 \%$ and $61.76 \%$.

Next, calculate the state transition probabilities in 2030 , 2040, and 2050 under different scenarios based on Markov theory, and the results are shown in Table 3. Combining the above prediction results of clean energy development potential, the state transition probability matrix is used to calculate the clean energy development potential of the transportation sector, construction sector and industry sector under different scenarios in 2030, 2040 and 2050. The calculation results are shown in Figure 3.
Table 3. Probability of state transition of sector path for clean energy development.

\begin{tabular}{ccccccccccc}
\hline \multicolumn{3}{c}{ Industry sector } & \multicolumn{3}{c}{ Construction sector } & \multicolumn{3}{c}{ Transportation sector } \\
& $\begin{array}{c}\text { Scen } \\
\text { ario } \\
\text { A }\end{array}$ & $\begin{array}{c}\text { Scen } \\
\text { ario } \\
\text { B }\end{array}$ & $\begin{array}{c}\text { Scen } \\
\text { ario } \\
\text { C }\end{array}$ & $\begin{array}{c}\text { Scen } \\
\text { ario } \\
\text { A }\end{array}$ & $\begin{array}{c}\text { Scen } \\
\text { ario } \\
\text { B }\end{array}$ & $\begin{array}{c}\text { Scen } \\
\text { ario } \\
\text { C }\end{array}$ & $\begin{array}{c}\text { Scen } \\
\text { ario } \\
\text { A }\end{array}$ & $\begin{array}{c}\text { Scen } \\
\text { ario } \\
\text { B }\end{array}$ & $\begin{array}{c}\text { Scen } \\
\text { ario } \\
\text { C }\end{array}$ \\
\hline 20 & & & & & & & & & \\
30 & 0.617 & 0.598 & 0.571 & 0.015 & 0.017 & 0.018 & 0.029 & 0.031 & 0.034 \\
ye & & & & & & & & & \\
ar \\
20 & & & & & & & & & \\
40 & 0.551 & 0.542 & 0.536 & 0.017 & 0.019 & 0.020 & 0.035 & 0.037 & 0.038 \\
ye & & & & & & & & & \\
ar \\
20
\end{tabular}

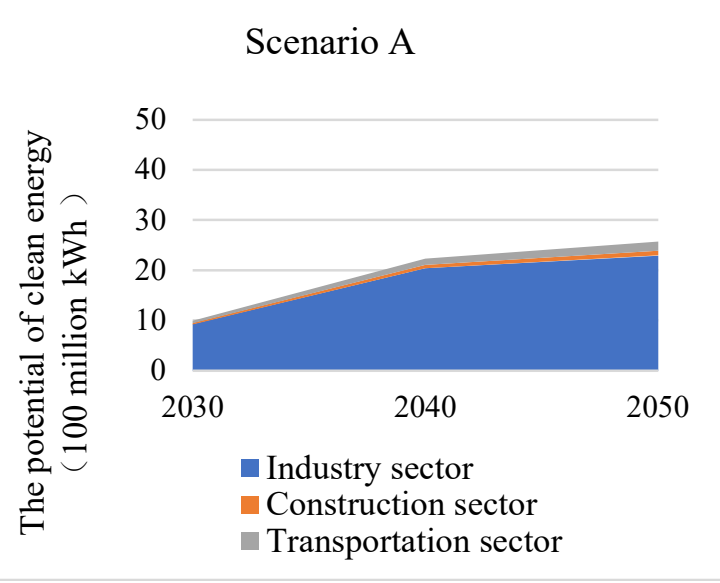

(a) Estimation results of clean energy potential of subpath under scenario A.

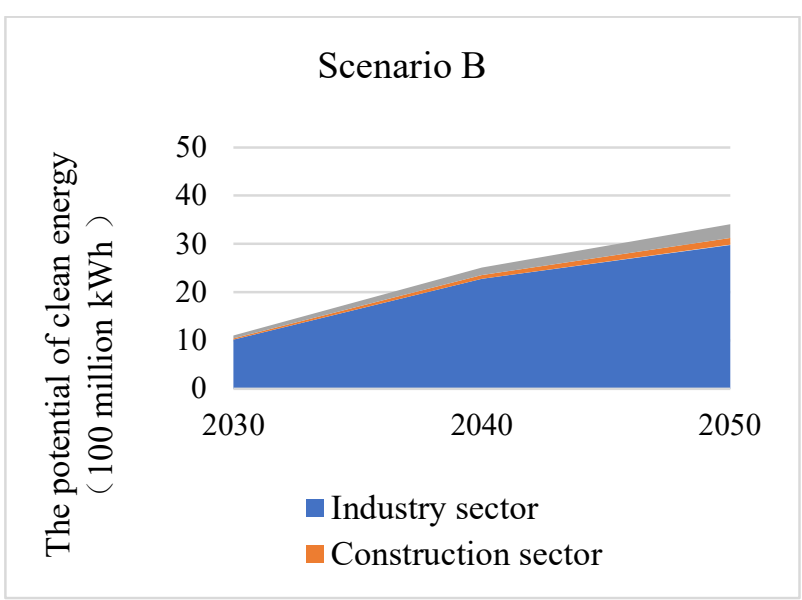

(b) Estimation results of clean energy potential of subpath under scenario B. 


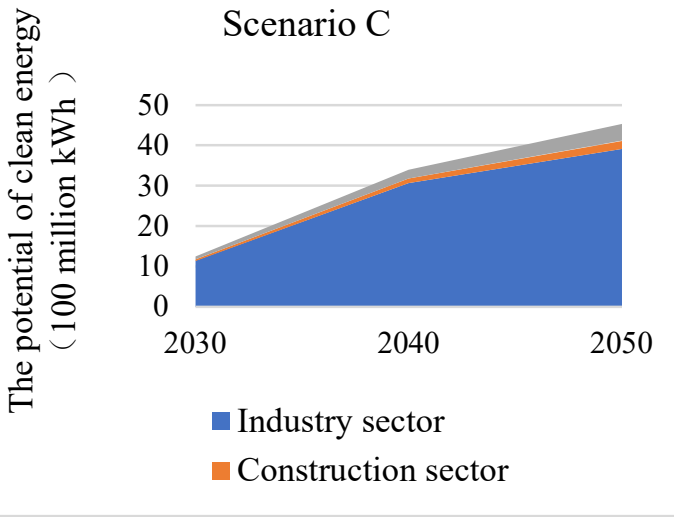

(c) Estimation results of clean energy potential of subpath under scenario C.

Figure 3. Calculation of the development potential of clean energy in the industry, construction and transportation sectors under various scenarios.

It can be seen from Figure 3 that, horizontally, the development potential of clean energy has shown a substantial growth trend under the influence of factors such as policy support and technological progress. In scenario A, the clean energy development potential of the industry, construction, and transportation sectors will reach 926 million $\mathrm{kWh}, 23$ million $\mathrm{kWh}$, and 44 million $\mathrm{kWh}$ respectively in 2030; they will reach 2.0387 million $\mathrm{kWh}, 80$ million $\mathrm{kWh}$, and 155 million $\mathrm{kWh}$ respectively in 2040; they will reach 2.295 billion $\mathrm{kWh}, 94$ million $\mathrm{kWh}$, and 179 million $\mathrm{kWh}$ respectively in 2050.Compared with scenario A, the clean energy development potential of the industry, construction, and transportation sectors under scenario B will increase by $9.82 \%, 26.08 \%$, and $20.45 \%$ respectively in 2030 ; increase by $11.6 \%, 26.8 \%$, and $20 \%$ respectively in 2040 ; increase by $29.9 \%, 55 \%$, and $51.22 \%$ respectively in 2050.In scenario C, the clean energy development potential of the industry, construction, and transportation sectors will reach $12.33 \%, 24.56 \%$, and $29.03 \%$ respectively in 2030; they will reach $34.7 \%, 42.8 \%$, and $39.3 \%$ respectively in 2040 ; they will reach $31.15 \%$, $43.76 \%$, and $46.6 \%$ respectively in 2050 .

\section{Conclusion}

From a vertical perspective, in the current stage of clean energy development, the main alternative areas are concentrated in the industry sector, and electric energy replaces scattered coal, coal-fired boilers and other areas with relatively mature technologies; secondly, in the transportation sector, electric motors replace internal combustion engines, realizing electric vehicles instead of fuel vehicles; finally, in the construction sector, it is mainly reflected in the combination of cold and hot spots and smart energy management systems. It is recommended to introduce subsidy policies related to clean energy development. On the one hand, it can improve the economics of electric energy substitution, and effectively guide the society to choose low-carbon, high-efficiency clean energy to replace traditional energy.
On the other hand, it is recommended to increase the research and development of electric energy substitution related technologies in transportation, industry, construction and other sectors to accelerate the improvement of the technical level, so as to enhance the development potential of clean energy.

\section{Acknowledgement}

This study is funded by the science and technology project of SGCC (Research on key technologies and modes of efficient operation of integrated energy system considering P2X and industry coupling, SGZJSX00XTJS2001473)

\section{References}

1. Fang Guochang, Wang Li, Gao Zhengye. Discussion on the coupling mechanism of energy internet and energy transition and identification of influencing factors[J]. Coal Economic Research, 2020, 40(11): 10-17.

2. Gao Zhiyuan,Zhang Tao,Zhao Lei,Zhang Jing,Hu Yuou,Cao Yang.Design of Priority Substitution Rules for Clean Energy Marketization and Related Analysis[J].Electric Power Automation Equipment, 2021,41(08):105-110.

3. Wang Huan,Ma Bing,Jia Lingxiao, Yu Yang,Hu Jiaxiu,Wang Wei.The role of key minerals in the clean energy transition under the goal of carbon neutrality, supply and demand analysis and recommendations[J/OL].Chinese Geology:1-19 [2021- 10-29].

http://kns.cnki.net/kcms/detail/11.1167.P.20211025. 1627.002.html.

4. Jiao Yuping, Cai Yu. An Analysis of China-Latin America Clean Energy Cooperation in the Context of Energy Transition[J/OL].Latin American Studies:114[2021-10-29].http://kns.cnki.net/kcms/detail /11.1160.C.20211015.2133.002.html.

5. China will accelerate the development and utilization of clean energy[J].Henan Science and Technology, 2021,40(09):2.

6. Hu Ming. Promote energy coupling development and break through industry energy barriers[N]. China Energy News, 2020-12-14(004).

7. Xingjunxia,Chen Yujie,Yao Hongyi.Analysis of Qinghai Province's Economic Growth and Energy Consumption Based on IPAT Model[J].Bohai Economic Outlook,2020(05):90-91.

8. Masao Nagasawa. Markov Processes and Quantum Theory[M].:2021-07-15. 